\title{
AEROSOL OPTICAL PROPERTIES RETRIEVED FROM THE FUTURE SPACE LIDAR MISSION ADM-AEOLUS
}

\author{
Pauline Martinet $^{1}{ }^{1 *}$, Thomas Flament ${ }^{1}$ and Alain Dabas ${ }^{1}$
}

${ }^{1}$ CNRM UMR 3589, Meteo FRance and CNRS, France, * pauline.martinet@meteo.fr

\begin{abstract}
The ADM-Aeolus mission, to be launched by end of 2017, will enable the retrieval of aerosol optical properties (extinction and backscatter coefficients essentially) for different atmospheric conditions. A newly developed feature finder (FF) algorithm enabling the detection of aerosol and cloud targets in the atmospheric scene has been implemented. Retrievals of aerosol properties at a better horizontal resolution based on the feature finder groups have shown an improvement mainly on the backscatter coefficient compared to the common $90 \mathrm{~km}$ product.
\end{abstract}

\section{INTRODUCTION}

The space-based Doppler mission AEOLUS of the European Space Agency expected to be launched towards end 2017 will be the first High-Spectral Resolution Lidar (HSRL) in space. Operating in the UV the HSRL capability is implemented by directing the backscattered light through 3 successive interferometers. The sensor behind the first one is dedicated to sensing particulate backscatter, its signal is called "Mie signal" and the sum of signals from the other two sensors is called "Rayleigh signal". These signals both contain contributions from molecular and particulate backscatter that are later seperated by computing a "cross talk correction". The system is primarily designed for the measurement of winds, but the HSRL capability enables the measurement of the particulate backscatter and extinction coefficients without any a priori assumption on the aerosol type. The level-2A
(L2A) processor has been developed to retrieve vertical profiles of optical properties in 24 bins from the ground up to $30 \mathrm{~km}$ with a vertical resolution varying from $250 \mathrm{~m}$ to $2 \mathrm{~km}$. The extinction coefficient is then derived through an iterative scheme while the backscatter coefficient is directly derived from the ratio of the pure particulate to molecular signals (more details can be found in [2]).

The ability of the L2A to retrieve clouds and aerosols properties over a $90-\mathrm{km}$ horizontal scale has already been demonstrated. A good retrieval of the overall shape of aerosol and cloud layers (see Fig. 1) with a better accuracy on the backscatter coefficient was shown in [1].

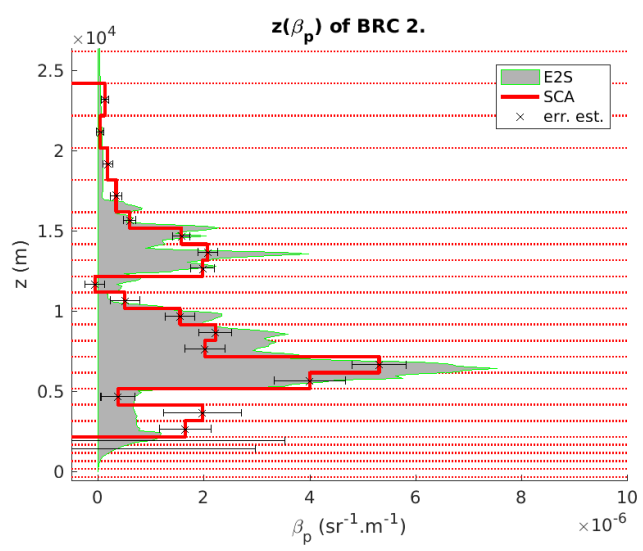

Figure 1: Vertical profile of L2A backscatter coefficients retrieval (red line) compared to the true expected profile (grey) and estimated retrieval errors (horizontal black lines). $90 \mathrm{~km}$ horizontal scale product

In order to improve the horizontal resolution of the retrieval and provide an aerosol product at a finer scale than $90 \mathrm{~km}$, a feature finder algorithm has been developed. It identifies homo- 
geneous aerosol and cloud targets on which the lidar signal is accumulated in order to obtain a sufficient signal to noise ratio (SNR).

\section{METHODOLOGY}

The feature finder developed for ADM-Aeolus is largely inspired by the EarthCare algorithm following the study of [3]. The algorithm is based on the assumption that both clear-sky and cloudy or aerosol loaded Mie signals as well as background noise follow a Gaussian distribution. If we consider a given signal level as a lower threshold, the part of the clear sky signal distribution above the threshold (red area in Fig. 2 ) is a false-alarm probability, i.e. the probability that the signal level in a clear sky pixel actually exceeds the threshold. The part of the particle-loaded distribution that falls below the threshold (dark green area in Fig. 2) is the probability of missing detection.

The probability of detection on the Mie channel $P_{M i e}$, i.e the bright green area, can be expressed by:

$$
P_{\text {Mie }}=1-\frac{1}{2} \operatorname{erfc}\left(\frac{S-\delta S}{\sqrt{2} \delta S}\right)
$$

with $S$ the signal level in a particle contaminated bin, $\delta S$ the noise level, and $\operatorname{erfc}$ the complementary error function.

The expression of the SNR is then used to simplify equation 1 :

$$
S N R=\frac{S}{\delta S}
$$

into:

$$
P_{M i e}=1-\frac{1}{2} \operatorname{erfc}\left(\frac{S N R-1}{\sqrt{2}}\right)
$$

The complementary erf $c$ function being monotonic, applying a threshold on $P_{M i e}$ is the same as applying a threshold on the Mie SNR. From a database of simulated scenarios, the SNR value enabling a high detection score has been determined.

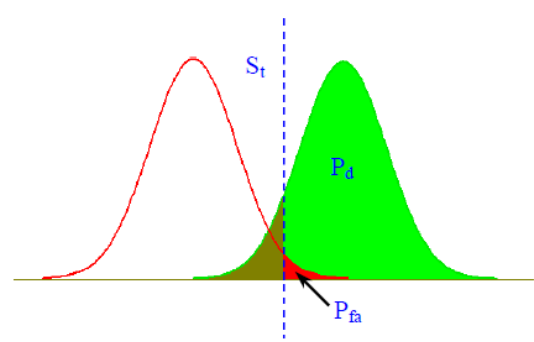

Figure 2: Probability of detection with the distribution for clear sky as a red line and the distribution for the particle-loaded signal as the green area. Figure extracted from the CALIOP ATBD described in [4].

\section{RESULTS}

\subsection{Feature Finder performance}

An End-to-End simulator (E2S) developed by ESA to simulate the lidar signals (Rayleigh and Mie channels) received by ADM-Aeolus has been used to simulate simple to more complex atmospheric scenes: standard boundary layer aerosols, additional thin and opaque clouds, real scenes observed during the LITE (Lidar Inspace Technology Experiment) experiment. By comparing the aerosol/cloud mask computed by the feature finder to the "true" E2S mask (input particulate backscatter coefficient larger than $10-6 \mathrm{~m}^{-1} \mathrm{sr}^{-1}$ ), the expected accuracy of the detection has been evaluated.

Figure 3 shows the feature finder performance on two LITE scenarios: the top panel shows a complex case with thin high clouds and middle level opaque clouds attenuating most of the signal (LITE C)whereas the bottom panel shows a simple case with thin cirrus clouds on top of boundary layer aerosols (LITE A). Particle contaminated pixels well detected by the feature finder are shown in yellow, false alarm in light green, missing aerosol in blue and clearsky pixels in dark blue. White pixels represent pixels for which a cloud detection is not possible due to the low transmittance of the lidar 
signal through the upper clouds (two-way transmittance smaller than 0.1). Taking into account the whole scene, a good detection of $72 \%$ with a false alarm rate of $4 \%$ was obtained for LITE C. The good detection rate is increased up to $83 \%$ if we only consider the atmosphere above the boundary layer in which the lidar signal is probably too noisy to reliably retrieve optical properties at a small scale. For LITE A, a good detection of $45 \%$ is shown for the whole scene but this rate is increased to $86 \%$ when considering the upper part of the atmosphere (above 3 $\mathrm{km})$ corresponding to the cirrus clouds. The developed FF algorithm is thus able to detect high level aerosol and clouds with a very high degree of good detection. However, the detection of boundary layer aerosol is still challenging due to the low noise level.

\subsection{Group retrievals}

In order to evaluate the capability of the L2A processor to retrieve aerosol properties at a finer horizontal scale compared to the $90 \mathrm{~km}$ accumulation length, aerosol retrievals have been performed after averaging lidar signals on small groups identified by the feature finder. These retrievals are then compared to the true E2S properties averaged on the same horizontal features. In order to emphasize the improvement in aerosol properties brought by an increased horizontal resolution, the retrievals at a $90 \mathrm{~km}$ horizontal length scale are also compared to the E2S averaged at the same length as used for the feature finder retrievals. We then compute the following ratio:

$$
R=\log \left(\frac{a b s\left(a e r_{90 k m}-a e r_{E 2 S, F F}\right)}{a b s\left(a e r_{F F}-a e r_{E 2 S, F F}\right)}\right)
$$

where $\operatorname{aer}_{F F}$ represents the aerosol retrieval (extinction or backscatter) accumulating the lidar signal on features detected by the algorithm, $\operatorname{aer}_{E 2 S, F F}$ represents the aerosol properties used in the E2S after accumulation on feature de-
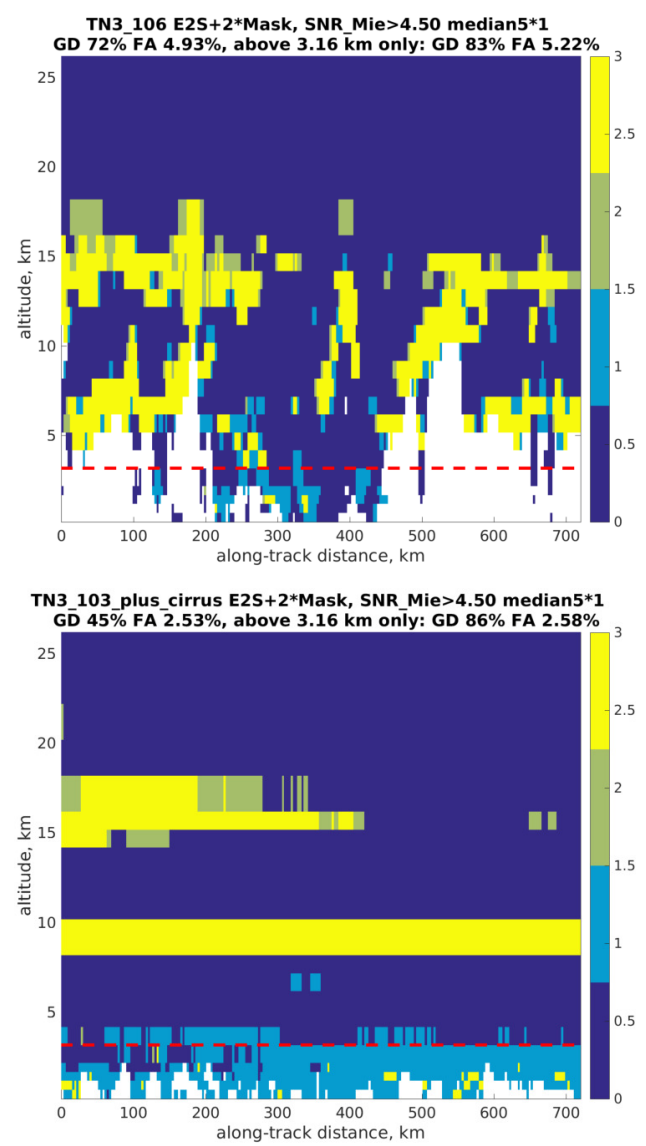

Figure 3: Feature Finder performance of good detection (yellow pixels), false alarm (green pixels), no detection (light blue) for the LITE C scenario with complex heterogeneous clouds (top panel) and the LITE A scenario with homogeneous high thin clouds (bottom panel).

tected by the algorithm and $a e r_{90 \mathrm{~km}}$ the aerosol retrievals performed by accumulating the lidar signal over $90 \mathrm{~km}$. Figure 4 shows this ratio for the complex LITE C scenario. Values above zero represent an improvement of the retrievals when using a smaller horizontal length, around zero similar performance is found, below zero the $90 \mathrm{~km}$ products leads to more consistent results with the E2S inputs. Both for the extinction and the backscatter coefficients, only few pixels show a degradation of the aerosol product if the lidar signal is accumulated on a finer 
scale. These pixels probably correspond to a strong attenuation of the lidar signal but this needs to be confirmed with a thorough evaluation. This first result demonstrates the robustness of the algorithm used to retrieve aerosol properties. For very long group, the performance is equivalent to the $90 \mathrm{~km}$ product as expected but a significant improvement is shown for the backscatter coefficient on small features below targets not attenuating too much the signal.
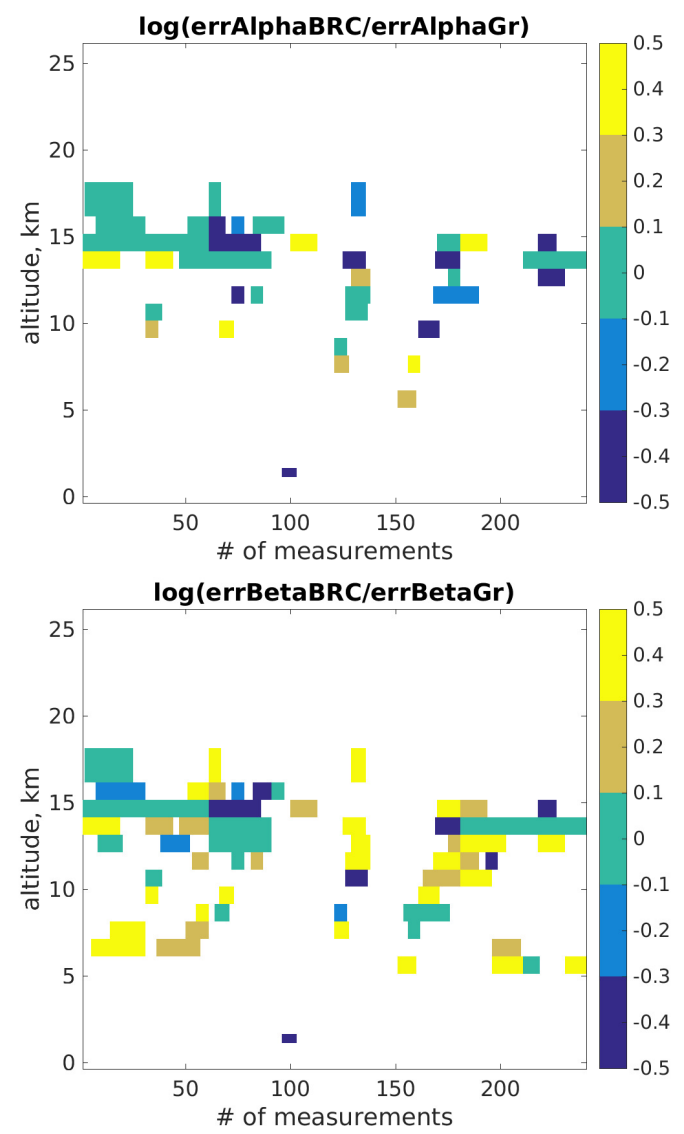

Figure 4: Ratio of the retrieval errors at a small horizontal scale to the retrieval errors at a $90 \mathrm{~km}$ accumulation length for the extinction coefficient (top panel) and backscatter coefficient (bottom panel). A logarithm scale is used

\section{CONCLUSIONS}

ADM-Aeolus has demonstrated its capability to fill the gap between the CALIPSO and EarthCare missions by retrieving aerosol and backscatter coefficients with a good accuracy. The newly developed feature finder algorithm has shown encouraging results to provide users with finer scale products in the case of heterogenous targets when the lidar signal is not strongly attenuated. The CAL/VAL activities are now in preparation in the view of the future satellite launch.

\section{ACKNOWLEDGEMENTS}

The work described in the paper was funded by the European Space Agency in the frame of the AEOLUS mission. We would like to thank ESA team - namely Frank de Bruin, Anne Straume, Thomas Kanitz - and the other members of the level 1/level 2A development teams for their advice, support and feedbacks.

\section{References}

[1] Martinet, P., Dabas, A., Lever, V., Flamant , P., Huber, D., EPJ Web of Conferences, 119, 04001, DOI: http://dx.doi.org/10.1051/epjconf/201611904001

[2] Flamant, P., Cuesta, J., Denneulin, M.-L., Dabas, A. and Huber, D., 2008: ADMAeolus retrieval algorithms for aerosol and cloud products. Tellus, 60A, 273-288

[3] Van Zadelhoff, G.-J. and Donovan, D., 2009: A potential FeatureMask algorithm for the EarthCARE lidar. 8th International Symposium on Tropospheric Profiling, ISBN 97890-6960-233-2

[4] Vaughan, M., Winker,D.M., Powell, K.A, 2005: CALIOP Algorithm Theoretical Baseline Document, Part 2: Feature Detection and Layer Properties Algorithms, PC-SCI202 Part 2. 